\title{
Genome Resource of Barley Bacterial Blight and Leaf Streak Pathogen Xanthomonas translucens pv. translucens strain UPB886
}

\author{
Verónica Roman-Reyna, ${ }^{1,2}$ Emily K. Luna, ${ }^{3}$ Céline Pesce, ${ }^{4,5,6}$ Taca Vancheva, ${ }^{4,5}$ Christine Chang, ${ }^{7}$ \\ Janet Ziegle, ${ }^{7}$ Claude Bragard, ${ }^{5}$ Ralf Koebnik, ${ }^{4}$ Jillian M. Lang, ${ }^{3}$ Jan E. Leach, ${ }^{3}$ and \\ Jonathan M. Jacobs ${ }^{1,2, \dagger}$ \\ ${ }^{1}$ Department of Plant Pathology, The Ohio State University, Columbus, OH, U.S.A. \\ ${ }^{2}$ Infectious Disease Institute, The Ohio State University, Columbus, OH, U.S.A. \\ ${ }^{3}$ Department of Bioagricultural Sciences and Pest Management, Colorado State University, Fort Collins, \\ CO, U.S.A. \\ ${ }^{4}$ IRD, Cirad, Université de Montpellier, IPME, Montpellier, France \\ ${ }^{5}$ Earth \& Life Institute, Université Catholique Louvain-la-Neuve, Louvain-la-Neuve, Belgium \\ ${ }^{6}$ Department of Molecular, Cellular, and Biomedical Sciences, University of New Hampshire, Durham, \\ $\mathrm{NH}$, U.S.A. \\ ${ }^{7}$ Pacific Biosciences, Menlo Park, CA, U.S.A.
}

\section{Abstract}

Xanthomonas translucens pv. translucens causes bacterial leaf streak and bacterial blight diseases of barley. This pathogen limits barley production globally but remains understudied, with limited genomic resources. To better understand the biology of this $X$. translucens subgroup, we sequenced the complete genome of the $X$. translucens $\mathrm{pv}$. translucens strain UPB886.

\section{Genome Announcement}

The plant-pathogenic bacterial species Xanthomonas translucens cause diseases of cereals, grasses, and even some dicots (Facelli et al. 2009; Jones et al. 1916). The pathovar translucens within $X$. translucens is most often associated with barley infection. $X$. translucens pv. translucens is not a wheat pathogen but has been previously isolated from wheat leaves (Bragard et al. 1997; Curland et al. 2018). X. translucens pv. translucens has been reported on all continents and is genetically diverse, comprising three subgroups $(A, B$, and $C$ ) based on phylogeny of concatenated housekeeping genes (Curland et al. 2018). Here, we describe the complete genome sequence of the $X$. translucens pv. translucens group $C$ strain UPB886 for a better comparative understanding of $X$. translucens host adaptation and biology.

X. translucens pv. translucens UPB886 was isolated from barley in Iran in 1990 (Alizadeh et al. 1995). We validated that strain UPB886 has pathogenic virulence on barley but not on spring wheat (pathogenicity test not shown). DNA was extracted by a standard phenol/ chloroform method (Booher et al. 2015). Strain UPB886 genomic DNA was sequenced using long-read, single-molecule real-time sequencing and assembled using HGAP v4 (PacBio, Menlo Park, CA, U.S.A.). Functional annotation of the two assembled contigs was done using Prokka v1.13.3, and secreted proteins were predicted based on SignalP algorithm as part of Prokka analysis (Petersen et al. 2011; Seemann 2014). Whole-genome comparison based on average nucleotide identity confirmed that this strain belonged to the $X$. translucens pv. translucens subgroup $C$ and had a high degree of synteny with other $X$. trans/ucens genomes (Table 1) (data not shown) (Curland et al. 2018; Rodriguez-R and Konstantinidis 2016).

${ }^{\dagger}$ Corresponding author: J. M. Jacobs; jacobs.1080@osu.edu

The author(s) declare no conflict of interest.

Accepted for publication 3 August 2019.

\section{Funding}

Funding was provided by the United States Department of Agriculture (USDA) National Institute of Food and Agriculture (NIFA) award number 2018-67013-28490 through the joint National Science Foundation-NIFA Plant Biotic Interactions Program to J. M. Jacobs, J. E. Leach, and J. M. Lang and a USDA-NIFA postdoctoral fellowship award number 2017-6701226116, a Fulbright Scholar Award to Belgium, to J. M. Jacobs. This article is based upon work from COST Action CA16107 EuroXanth, supported by COST (European Cooperation in Science and Technology).

\section{Keywords}

bacteria, barley, genome, TALE, Xanthomonas translucens 
The $X$. translucens pv. translucens UPB886 genome encoded typical virulence factors found in Xanthomonas spp. The genome has 15 genes encoding extracellular plant cell-walldegrading enzymes, 7 genes involving in copper resistance cluster, and 21 genes related to the type II secretion system. Type III secretion is critical for $X$. translucens pv. translucens pathogenesis (Pesce et al. 2017), and the strain UPB886 genome encoded 41 conserved type III-secreted proteins including transcriptional activator-like effectors (TALEs). The strain UPB886 genome had five TALEs (Table 2); three were shared with other $X$. translucens and assigned to classes TaICV, TaIDD, and TaICT. The remaining two TALEs were unique to strain UPB886 and belong to classes TalAJ and TalEU (Falahi Charkhabi et al. 2017; Grau et al. 2016; Peng et al. 2016). X. translucens pv. translucens UPB886 provides a genomic resource to advance $X$. translucens research. This resource will allow further genetic diversity

Table 1. Complete genomes summary of Xanthomonas translucens pvs. undulosa and translucens

\begin{tabular}{|c|c|c|c|c|c|c|c|c|c|}
\hline \multirow[b]{3}{*}{ Strain $^{a}$} & \multirow{2}{*}{\multicolumn{2}{|c|}{ Isolation source }} & \multicolumn{3}{|c|}{ Genome analysis } & \multicolumn{3}{|c|}{ Annotation } & \multirow[b]{3}{*}{$A N I^{d}$} \\
\hline & & & \multirow{2}{*}{$\begin{array}{l}\text { Genome size } \\
\text { (bp) }\end{array}$} & \multirow[b]{2}{*}{ Contigs } & \multirow{2}{*}{$\begin{array}{c}\text { GC content } \\
(\%)\end{array}$} & \multirow[b]{2}{*}{ CDS $^{b}$} & \multirow[b]{2}{*}{ TALEs $^{c}$} & \multirow{2}{*}{$\begin{array}{l}\text { Total secreted } \\
\text { proteins }\end{array}$} & \\
\hline & Year & Country & & & & & & & \\
\hline \multicolumn{10}{|l|}{$\begin{array}{l}X . \text { translucens pv. } \\
\text { translucens }\end{array}$} \\
\hline $\begin{array}{l}\text { UPB886 } \\
\text { SAMN11831831 }\end{array}$ & 1990 & Iran & $4,674,364$ & 2 & 67.9 & 3,889 & 5 & 547 & 100 \\
\hline $\begin{array}{l}\text { DSM18974 } \\
\text { NZ_LT604072.1 }\end{array}$ & 1933 & $\begin{array}{l}\text { United } \\
\text { States }\end{array}$ & $4,715,357$ & 1 & 67.7 & 3,957 & 8 & 563 & 99 \\
\hline \multicolumn{10}{|l|}{$\begin{array}{l}\text { X. translucens pv. } \\
\text { undulosa }\end{array}$} \\
\hline $\begin{array}{l}\text { XT4699 } \\
\text { NZ_CP008714.1 }\end{array}$ & 1999 & $\begin{array}{l}\text { United } \\
\text { States }\end{array}$ & $4,561,137$ & 1 & 68.1 & 3,528 & 8 & 531 & 98 \\
\hline $\begin{array}{l}\text { ICMP11055 } \\
\text { NZ_CP009750.1 }\end{array}$ & 1983 & Iran & $4,761,583$ & 1 & 67.8 & 3,953 & 7 & 540 & 98 \\
\hline
\end{tabular}

a Lines indicate species, strain, and NCBI reference number.

b Coding sequence.

c Transcription activator-like effectors.

d Average nucleotide identity (ANI) is relative to UPB886.

Table 2. RVD sequences of Xanthomonas translucens pvs. undulosa and translucens

\begin{tabular}{|c|c|c|c|}
\hline $\begin{array}{l}\text { TALE } \\
\text { classes }^{a}\end{array}$ & Strain ${ }^{\mathbf{b}}$ & RVD sequence alignment & Genome location:strand \\
\hline TalAJ & UPB886 & NN HD NG NI HD HD HD & $4,505,717-4,508,111:-1$ \\
\hline \multirow[t]{4}{*}{ TalCT } & DSM18974 & NN HD HD HD NI NI NI NN HD HD NN NN NI NN HD & $671,336-674,561: 1$ \\
\hline & UPB886 & NN HD HD HD NI NI NI HN HD HD NN NN NI NN HD & $4,482,729-4,485,960:-1$ \\
\hline & XT4699 & HN HD HD HD NI NI NI HN HD HD NH NN NI NN HD & $3,253,498-3,256,723:-1$ \\
\hline & ICMP11055 & HN HD HD HD NI NI NI HN HD HD NN NN NI NN HD & $1,470,456-1,473,681: 1$ \\
\hline TalCU & DSM18974 & NG HD HD HN NG NI HG HG HD ND NN NN NI NH QD & $3,565,727-3,568,955:-1$ \\
\hline \multirow[t]{2}{*}{ TalCV } & DSM18974 & NG NN HD HD NN NI HG HD ND HG NI NN HD & $3,562,396-3,565,408:-1$ \\
\hline & UPB886 & NG NN HD HD NN NI HG HD ND HG NI NN HD & $1,623,874-1,626,892: 1$ \\
\hline TalCW & DSM18974 & NN NI HN HD NI NH NG HN HD HD HD NI QD & $660,311-663,329: 1$ \\
\hline TalCX & DSM18974 & NNHDNGNI HNKG NI HDNINH NG NN HD HDNI NN NI HD QD & $663,647-667,295: 1$ \\
\hline TalCY & DSM18974 & NI NG HN NN HD NG ND NK QD NH QD & $668,858-671,207: 1$ \\
\hline \multirow[t]{3}{*}{ TalCZ } & XT4699 & NH NN HD NN HD NH HD YK NG NH Y* HD NN NI NG QD NH NN & $2,669,985-2,673,321:-1$ \\
\hline & DSM18974 & HD NN HD NH HD YK NG NH Y* HD NN NI NG QD NH NN HD NN & $1,995,271-1,998,607: 1$ \\
\hline & ICMP11055 & HD NH HD YK NG NH $Y^{*}$ HD NN NI NG Q & $2,119,325-2,122,661:-1$ \\
\hline \multirow[t]{3}{*}{ TalDA } & DSM18974 & NN HD NG NG NG NN YK NG HD NG NG ND NG HD NH HD & $627,243-630,573: 1$ \\
\hline & XT4699 & HD YD NI NG NG NN YK NG HD NG NG ND NG QD NH HD & $584,465-587,645: 1$ \\
\hline & ICMP11055 & HD YD NI NG NG NN YK NG HD NG NG ND NG QD NH HD & $627,709-630,889: 1$ \\
\hline TalDB & XT4699 & NN HD NG HD HD HN NF NI NH HD HD HD HN HN HD & 1,991,439-1,994,667:-1 \\
\hline TalDC & XT4699 & NN NG HD HD HD KG NN Y* NG HD HD QD HN & $3,257,061-3,260,076:-1$ \\
\hline \multirow[t]{3}{*}{ TalDD } & UPB886 & NN QD NG NN HN KG NI HD NI NH NG HN HD HD NI NN HD & $4,486,088-4,489,529:-1$ \\
\hline & XT4699 & NN HD NG NN HN KG NI HD NI NN HD HN HD HD NI HN HD QD & $605,042-608,582: 1$ \\
\hline & ICMP11055 & NN HD NG NN HN KG NI HD NI HN HD HN HD $Y^{*}$ NG HD HD HN & $648,160-651,691: 1$ \\
\hline TalDE & XT4699 & NN HD NG NN HN HN NI NI NI NH NN HD NN NH HD HD & $2,857,126-2,860,465:-1$ \\
\hline \multirow[t]{2}{*}{ TalDF } & XT4699 & HD HN HN HD NH NH HG HD KG NN $Y^{\star} N G$ HD HD HN & $2,861,842-2,865,061:-1$ \\
\hline & ICMP11055 & HD HN HN HD NH NH HG HD KG NN Y* NG HD HD HN & $1,918,202-1,921,427: 1$ \\
\hline TalEV & ICMP11055 & NN HD NG HD HD HG HD KG NN Y* NG NG HD HD QD HN & $1,924,847-1,928,171: 1$ \\
\hline TalEW & ICMP11055 & NN HD NG HD NG HD HD HG HD KG NN KG HD HN QD HN & $1,466,788-1,470,118: 1$ \\
\hline TalEX & UPB886 & NI NG HN NK HD NH HN HD HD HD HD QD & $2,350,128-2,353,041:-1$ \\
\hline
\end{tabular}

a Transcriptional activator-like effector (TALE) classes based on annoTALE (Grau et al. 2016).

b Xanthomonas translucens strains. 
studies to understand the mechanisms underlying disease development, improve tools for diagnostics, and assist barley breeding programs.

The whole genome was deposited at GenBank under BioSample accession number

SAMN11831831.

\section{Literature Cited}

Alizadeh, A., Barrault, G., Sarrafi, A., Rahimian, H., and Albertini, L. 1995. Identification of bacterial leaf streak of cereals by their phenotypic characteristics and host range in Iran. Eur. J. Plant Pathol. 101:225-229.

Booher, N. J., Carpenter, S. C. D., Sebra, R. P., Wang, L., Salzberg, S. L., Leach, J. E., and Bogdanove, A. J. 2015. Single molecule real-time sequencing of Xanthomonas oryzae genomes reveals a dynamic structure and complex TAL (transcription activator-like) effector gene relationships. Microb. Genomics 1:e000032.

Bragard, C., Singer, E., Alizadeh, A., Vauterin, L., Maraite, H., and Swings, J. 1997. Xanthomonas translucens from small grains: Diversity and phytopathological relevance. Phytopathology 87:1111-1117.

Curland, R. D., Gao, L., Bull, C. T., Vinatzer, B. A., Dill-Macky, R., Van Eck, L., and Ishimaru, C. A. 2018. Genetic diversity and virulence of wheat and barley strains of Xanthomonas translucens from the Upper Midwestern United States. Phytopathology 108:443-453.

Facelli, E., Taylor, C., Williams, N. M., Emmett, R. W., Sedgley, M., Joyce, C. K., and Scott, E. S. 2009. Location of Xanthomonas translucens in pistachio trees. Australas. Plant Pathol. 38:584-593.

Falahi Charkhabi, N., Booher, N. J., Peng, Z., Wang, L., Rahimian, H., ShamsBakhsh, M., Liu, Z., Liu, S., White, F. F., and Bogdanove, A. J. 2017. Complete genome sequencing and targeted mutagenesis reveal virulence contributions of Tal2 and Tal4b of Xanthomonas translucens pv. undulosa ICMP11055 in bacterial leaf streak of wheat. Front. Microbiol. 8:1488.
Grau, J., Reschke, M., Erkes, A., Streubel, J., Morgan, R. D., Wilson, G. G., Koebnik, R., and Boch, J. 2016. AnnoTALE: Bioinformatics tools for identification, annotation, and nomenclature of TALEs from Xanthomonas genomic sequences. Sci. Rep. 6: Article 21077.

Jones, L. R., Johnson, A. G., and Reddy, C. S. 1916. Bacterial blights of barley and certain other cereals. Science 44:432-433.

Peng, Z., Hu, Y., Xie, J., Potnis, N., Akhunova, A., Jones, J., Liu, Z., White, F. F., and Liu, S. 2016. Long read and single molecule DNA sequencing simplifies genome assembly and TAL effector gene analysis of Xanthomonas translucens. BMC Genomics 17: Article 21.

Pesce, C., Jacobs, J. M., Berthelot, E., Perret, M., Vancheva, T., Bragard, C., and Koebnik, R. 2017. Comparative genomics identifies a novel conserved protein, HpaT, in proteobacterial type III secretion systems that do not possess the putative translocon protein HrpF. Front. Microbiol. 8: 1177.

Petersen, T. N., Brunak, S., von Heijne, G., and Nielsen, H. 2011. SignalP 4.0: Discriminating signal peptides from transmembrane regions. Nat. Methods 8 : 785-786.

Rodriguez-R, L. M., and Konstantinidis, K. T. 2016. The enveomics collection: A toolbox for specialized analyses of microbial genomes and metagenomes. PeerJ Inc. https://peerj.com/preprints/1900/

Seemann, T. 2014. Prokka: Rapid prokaryotic genome annotation. Bioinformatics 30: 2068-2069. 